\title{
Autoregressive conditional kurtosis
}

\author{
Article
}

Accepted Version

Brooks, C., Burke, S. P., Heravi, S. and Persand, G. (2005) Autoregressive conditional kurtosis. Journal of Financial Econometrics, 3 (3). pp. 399-421. ISSN 1479-8417 doi: https://doi.org/10.1093/jjinec/nbi018 Available at https://centaur.reading.ac.uk/20558/

It is advisable to refer to the publisher's version if you intend to cite from the work. See Guidance on citing.

Published version at: http://dx.doi.org/10.1093/jifinec/nbio18

To link to this article DOI: http://dx.doi.org/10.1093/jjfinec/nbi018

Publisher: Oxford University Press

All outputs in CentAUR are protected by Intellectual Property Rights law, including copyright law. Copyright and IPR is retained by the creators or other copyright holders. Terms and conditions for use of this material are defined in the End User Agreement.

\section{www.reading.ac.uk/centaur}

\section{CentAUR}

Central Archive at the University of Reading

Reading's research outputs online 
This is a pre-copyedited, author-produced PDF of an article accepted for publication in the Journal of Financial Econometrics following peer review. The definitive publisher-authenticated version ( $C$. Brooks, S.P. Burke, S. Heravi and G. Persand, 'Autoregressive Conditional Kurtosis', Journal of Financial Econometrics, 3.3 (2005)) is available online at: http://jfec.oxfordjournals.org/content/3/3/399 


\title{
Autoregressive Conditional Kurtosis
}

\author{
Chris Brooks ${ }^{1}$, Simon P. Burke ${ }^{2}$, Saeed Heravi, ${ }^{3}$, Gita Persand ${ }^{4}$
}

The authors' affiliations are ${ }^{1}$ Corresponding author: Cass Business School, City of London, 106 Bunhill Row, London EC1Y 8TZ, UK, tel: (+44) 20704051 68; fax: (+44) 2070408881 41; e-mail: C.Brooks@city.ac.uk; ${ }^{2}$ School of Business, University of Reading ${ }^{3}$ Cardiff Business School, and ${ }^{4}$ Management School, University of Southampton. We would like to thank the Editor, Eric Renault, an Associate Editor, and two anonymous referees for useful comments on an earlier version of this paper. We would also like to thank seminar participants at the following UK universities for useful discussions: Kings College, London; Cass Business School; Reading; Essex; Bristol; Manchester. The usual disclaimer applies. 


\begin{abstract}
This paper proposes a new model for autoregressive conditional heteroscedasticity and kurtosis. Via a time-varying degrees of freedom parameter, the conditional variance and conditional kurtosis are permitted to evolve separately. The model uses only the standard Student's $t$ density and consequently can be estimated simply using maximum likelihood. The method is applied to a set of four daily financial asset return series comprising US and UK stocks and bonds, and significant evidence in favour of the presence of autoregressive conditional kurtosis is observed. Various extensions to the basic model are proposed, and we show that the response of kurtosis to good and bad news is not significantly asymmetric.
\end{abstract}

J.E.L. Classifications: C22, C51, C52

Keywords: conditional kurtosis, GARCH, fourth moment, fat tails, Student's t distribution

\title{
Introduction
}

It is an almost universally accepted stylised fact that asset returns are not normally distributed, following early research by Mandelbrot (1963), but rather that they are leptokurtic. This property implies that extreme market movements, in either direction, will occur with greater frequency in practice than would be predicted by the normal distribution. For example, a 5\% daily loss is observed to occur in equity markets approximately once every two years, while if returns were normally distributed, such a change would be expected only once in every one thousand years (Johansen and Sornette, 1999), given the estimated return variances. Clearly this is an important observation in finance since, under the normality assumption for returns, variance is widely used as a proxy for market risk. If however, asset returns are fat-tailed, this will lead to a systematic 
underestimate of the true riskiness of a portfolio, where risk is measured as the likelihood of achieving a loss greater than some threshold.

The standard ARCH and GARCH models introduced by Engle (1982) and Bollerslev (1986) respectively allow normally distributed disturbances to have time varying (conditional) variance. Such models are able to generate data with unconditionally fat tails, but not sufficiently fat to capture all of the observed unconditional leptokurtosis in returns series. Engle and Bollerslev (1986) explore the Gaussian model further, and although conditional kurtosis is not of direct interest to their study, they derive the conditional kurtosis forecasts from a $\operatorname{GARCH}(1,1)$ process as a function of the conditional variance. Their derivation is sufficient to illustrate a key point relevant to this paper: since the normal distribution is characterised entirely by its first two moments, the behaviour of the kurtosis is entirely determined by that of the variance.

The observation that GARCH models with normal disturbances cannot generate sufficient leptokurtosis to replicate that observed in actual data was in part the motivation for the study of Bollerslev (1987). He developed a more general model that allowed the disturbances to have a transformed $t$-distribution so that extreme values, occurring more commonly than under a normal distribution, may be accommodated. However, whilst such a model can lead to sufficiently fat tails to provide a realistic model for asset returns, the conditional kurtosis from such a model is tied to the conditional variance via a time-invariant degrees of freedom parameter. To introduce some notation, suppose that the conditional distribution of the series of interest, $y_{t}, t=1,2, \ldots, T$, is a transformed central $t$ with conditional mean $y_{t \mid t-1}$, variance $h_{t \mid t-1}$ and degrees of freedom $v$, 
and let $f_{v}\left(\varepsilon_{t} \mid \psi_{t-1}\right)$ denote the density function for $\varepsilon_{t}$ conditional upon all information available to time $t$-1. A GARCH-t model may thus be written

$$
\begin{aligned}
& y_{t}=\gamma_{0}+\varepsilon_{t} \quad, \quad \varepsilon_{t} \mid \psi_{t-1} \sim f_{v}\left(\varepsilon_{t} \mid \psi_{t-1}\right) \\
& h_{t \mid t-1}=\alpha_{0}+\alpha_{1} \varepsilon_{t-1}{ }^{2}+\alpha_{2} h_{t-1 \mid t-2}
\end{aligned}
$$

where $\gamma_{0}, \alpha_{0}, \alpha_{1}, \alpha_{2}$ are parameters to be estimated.

From a finance perspective, consideration of the higher moments of portfolio return distributions is important to ensure that investors make optimal decisions given their tolerances for risk and so that fund management or trading rule performance is correctly appraised. Research by Chunhachinda et al. (1997), for instance, suggests that the incorporation of moments higher than the second into the investor's portfolio decision causes a major change in the construction of the optimal combination of risky assets. Since higher moment deviations from normality are agreed to be non-negligible, there is no reason to suppose that they should be time-invariant, other than for simplicity, and allowing them to be time-varying may improve their approximation to the actual return distributions. Failure to consider moments higher than the second or assuming that those moments are time-invariant could also lead to avoidably high approximation error. Nelson (1996), for example, plots the standardised residuals exceeding 4 in absolute value from an EGARCH fit to daily S\&P 500 data, and finds that large residuals of either sign tend to bunch together through time. He argues that this finding implies evidence for time-varying kurtosis.

More recent research by Harvey and Siddique $(1999,2000)$ has proposed and employed a model that allows for time-varying conditional skewness, based upon a non-central Student's $t$ distribution. However, their approach does not model time-varying conditional kurtosis. We 
would argue that an examination of the conditional fourth moment is of importance given that leptokurtosis is almost universally observed in financial asset returns, irrespective of the frequency of observation (although excess kurtosis is seen to diminish somewhat with temporal and cross-sectional aggregation of returns). On the other hand, some asset return distributions for emerging market returns appear to be negatively skewed (implying a higher probability of negative returns than positive returns of the same order - see Bekaert, Erb, Harvey and Viskanta, 1998), while others are positively skewed. The degree and sign of the conditional skewness appears also to vary with sampling frequency and for given assets over time (see Harvey and Siddique, 1999, Figure 3B, for an illustration of the latter).

Fernandez and Steel (1998) introduce an alternative model for conditional skewness that can be applied to any continuous, unimodal and symmetric distribution. The method is then applied to a set of stock returns, although they examine only unconditional moments. Lambert and Laurent (2001) employ the Fernandez and Steel approach in the context of maximum likelihood estimation of a GARCH model. They do not, however, consider conditional third or fourth moments.

Hansen (1994) develops a general model for autoregressive conditional density estimation, centred on a skewed version of the Student's $t$ density. The skewed Student's $t$ is different from that of Harvey and Siddique (1999) and the skewness is achieved by modifying the density of the symmetric Student's $t$ distribution. Hansen allows the degree of freedom parameter to vary over time within prescribed bounds (2.1 to 30$)$ using a logistic function. Hansen's density collapses to the symmetric student $t$ when the asymmetry parameter is zero. Premaratne and Bera (2001) propose the use of a Pearson type IV distribution for the unconditional returns data, which is able 
to account for both asymmetry and extreme fat tails simultaneously, although they do not fully examine these in a conditional setting that allows the third and fourth moments to be timevarying. An alternative unconditional distribution that can account for both skewness and leptokurtosis is the skewed generalised $t$ distribution proposed by Theodossiou (1998). The various types of asymmetric conditional density function that are available for GARCH-type modelling are reviewed in Bond (2001).

In this paper, we develop a model for autoregressive conditional kurtosis that permits the kurtosis to develop over time in a fashion that is not fixed with respect to the variance. This is achieved via a central $t$-distribution with time-varying degrees of freedom, so that the variance, degrees of freedom and hence kurtosis all vary over time, in a manner determined by the data. It is this allowance of the degrees of freedom parameter to vary over time that permits the relaxation of the relationship between the variance and kurtosis. It is worth noting at the outset that such a set up with a central $t$ cannot produce asymmetries in the conditional return distribution, although incorporation of asymmetry terms in the conditional variance and conditional kurtosis equations can generate asymmetries in the unconditional return distribution. For the reasons above, and for its tractability and ease of subsequent model estimation, the restriction implied by the use of a central rather than a non-central $t$ may not be undesirable. As Premaratne and Bera (2001) note, the non-central $t$ involves the sum of an infinite series which enters the log-likelihood function and which makes computation exceedingly difficult. The Hansen (1994) approach allows for time-varying skewness but requires the imposition of several restrictions on the parameter values to permit estimation. These parameter restrictions are lifted in work by Jondeu and Rockinger (2000). Our model, by contrast, makes use of the central Student's $t$ distribution and can be viewed as a natural extension of Bollerslev (1987) that fits within the GARCH genre of models. 
We allow for asymmetries in the returns distributions via an indicator function employed in the conditional variance and conditional kurtosis equations.

The remainder of the paper is laid out as follows. Section 1 develops the univariate model for conditional kurtosis and discusses estimation issues, while Section 2 describes the data that we employ to illustrate the model's applicability. Section 3 discusses the univariate results and their interpretation. Finally, Section 4 concludes and offers suggestions for further research.

\section{Model Development}

\subsection{The Framework}

In order to obtain a model with freely varying conditional kurtosis as well as conditional variance, an approach based on that of Bollerslev (1987), but extended, is employed. Let $\varepsilon_{t}$, $t=1,2, \ldots, T$, be independently distributed as central Student's $t$ variates with $v_{t}$ degrees of freedom. Extending the work of Bollerslev (1987), consider a time varying transformation of $\varepsilon_{t}$, to result in a new process that may have any desired variance $h_{t}$ and kurtosis $k_{t}$. Let this be given by

$$
\varepsilon_{t}^{*}=\lambda_{t} \varepsilon_{t}
$$

where the $\varepsilon_{t}^{*}$ are the analogues of the disturbances of a $t$-GARCH model. We can state the timevarying transformation as a function of the conditional kurtosis and the conditional variance:

$$
\lambda_{t}=\left(\frac{k_{t} h_{t}}{2 k_{t}-3}\right)^{1 / 2}
$$


The conditional variance, $h_{t}=\mu_{2, t}$ and conditional fourth moment, $\mu_{4, t}$, are then given respectively by

$$
\begin{aligned}
& \mu_{2, t}=\lambda_{t}^{2} \frac{v_{t}}{v_{t}-2} ; \\
& \mu_{4, t}=\lambda_{t}^{4} \frac{3 v_{t}^{2}}{\left(v_{t}-2\right)\left(v_{t}-4\right)} .
\end{aligned}
$$

Equations (4) arise from the moment generating function for a central $t$, where all odd moments are by definition zero. Rearranging equation (4a) gives the time-varying transformation as a function of the conditional variance and the time-varying degrees of freedom

$$
\lambda_{t}=\left(\frac{h_{t}\left(v_{t}-2\right)}{v_{t}}\right)^{1 / 2}
$$

Defining $k_{t}=\mu_{4, t} / h_{t}^{2}$, and substituting (5) into (4b) gives the conditional kurtosis as a function of the degrees of freedom at time $t$ as

$$
k_{t}=\frac{3\left(v_{t}-2\right)}{\left(v_{t}-4\right)} .
$$

Equation (6) can be rearranged to give the degrees of freedom as a function of the conditional kurtosis

$$
v_{t}=\frac{2\left(2 k_{t}-3\right)}{k_{t}-3}
$$

The equations above show that the conditional variance and conditional kurtosis are not tied together in a fixed fashion and may thus vary freely over time, since $k_{t}$ is a function of $v_{t}$ only while $h_{t}$ also depends on $\lambda_{t}$. Since they are not directly functionally related, the terms $h_{t}$ and $k_{t}$ 
may be parameterised individually as desired. In order to estimate the parameters of these processes, note that the Jacobian of the transformation $\varepsilon_{t}^{*} / \lambda_{t}=\varepsilon_{t}$ is

$$
J=\frac{\partial \varepsilon_{t}}{\partial \varepsilon_{t} *}=\frac{1}{\lambda_{t}}
$$

Taking the Student's $t$ density for $\varepsilon_{t}$, substituting $\varepsilon_{t}^{*} / \lambda_{t}=\varepsilon_{t}$ and multiplying by the Jacobian gives the density of $\varepsilon_{t}^{*}$ :

$$
f\left(\varepsilon_{t}^{*}\right)=\frac{1}{\lambda_{t}} \frac{\Gamma\left[\left(v_{t}+1\right) / 2\right]}{\pi^{1 / 2} v_{t}^{1 / 2} \Gamma\left[v_{t} / 2\right]\left(1+\varepsilon_{t}^{* 2} / \lambda_{t}^{2} v_{t}\right)^{\left(v_{t}+1\right) / 2}} .
$$

Substituting for $\lambda_{t}$ in (9) and taking logarithms yields the log-likelihood for the $t^{\text {th }}$ observation (dropping constant terms):

$$
\begin{aligned}
l_{t}=\log \left(\Gamma\left[\left(v_{t}+1\right) / 2\right]\right)-\log \left(\Gamma\left[v_{t} / 2\right]\right) & -\frac{1}{2} \log \left(h_{t}\right)-\frac{1}{2} \log \left(v_{t}-2\right) \\
& -\frac{v_{t}+1}{2} \log \left(1+\frac{\varepsilon_{t}^{* 2}}{h_{t}\left(v_{t}-2\right)}\right)
\end{aligned}
$$

where $v_{t}$ is a function of $k_{t}$, as given by (6). Maximisation of the log-likelihood function, $\sum_{t=1}^{T} l_{t}$, yields the maximum likelihood estimates of all the parameters of the model.

Many specifications of the variance and kurtosis equations are conceivable, most obviously they may be of the typical GARCH type, namely,

$$
\begin{aligned}
& h_{t}=\alpha_{0}+\alpha_{1} \varepsilon_{t-1}^{* 2}+\alpha_{2} h_{t-1}, \\
& k_{t}=\beta_{0}+\beta_{1} \frac{\varepsilon_{t-1}^{* 4}}{h_{t-1}^{2}}+\beta_{2} k_{t-1},
\end{aligned}
$$


An even more general specification could be entertained, where feedback between the conditional kurtosis and conditional variance are allowed for. However, such a model would be highly parameterised and we therefore do not consider it further in this study.

To summarise, the model may be termed generalised autoregressive conditional heteroscedasticity and kurtosis (GARCHK), and may be described by the following equations.

$$
\begin{aligned}
& y_{t}=\gamma_{0}+\varepsilon_{t}^{*} ; \\
& \varepsilon_{t}^{*}=\lambda_{t} \varepsilon_{t}, \varepsilon_{t} \sim t_{v_{t}} \\
& h_{t}=\alpha_{0}+\alpha_{1} \varepsilon_{t-1}^{* 2}+\alpha_{2} h_{t-1} ; \\
& k_{t}=\beta_{0}+\beta_{1} \frac{\varepsilon_{t-1}^{*}}{h_{t-1}^{2}}+\beta_{2} k_{t-1} ; \\
& v_{t}=\frac{2\left(2 k_{t}-3\right)}{k_{t}-3} ; \\
& \lambda_{t}=\left(\frac{h_{t}\left(v_{t}-2\right)}{v_{t}}\right)^{1 / 2} .
\end{aligned}
$$

All parameters are estimated using quasi-maximum likelihood with the BFGS algorithm. From equation (6) above, there exists a degrees of freedom restriction,

$$
v_{t}>4
$$

generated by the requirement for the existence of a fourth moment. Equation (6) also implies that $k_{t} \rightarrow 3$ as $v_{t} \rightarrow \infty$ and $k_{t} \rightarrow \infty$ as $v_{t} \rightarrow 4$. In order to ensure that the estimated model is admissible, it is sufficient that $\alpha_{0}, \beta_{0}, \alpha_{1}, \beta_{1}>0, \alpha_{2}, \beta_{2} \geq 0, h_{t}>0 \forall t$, and $k_{t}>3 \forall t$. We did not have to physically impose these restrictions on the estimation process since they seemed to arise naturally 
from the data. The initialisations for the conditional variance and kurtosis series were set with every element equal to their unconditional values.

\subsection{Development of a Test for Autoregressive Conditional Kurtosis}

At first blush, it may appear sensible to attempt the formulation of test for autoregressive conditional kurtosis in a similar vein to Engle's $T R^{2}$ Lagrange Multiplier test for conditional heteroscedasticity, defining, $y_{t}=\frac{\hat{\varepsilon}_{t}^{4}}{\hat{h}_{t}^{2}}$, and regressing it on $p$ of its lagged values to test for autoregressive conditional kurtosis of order $p$. However, such an approach is inappropriate in the context of a student- $t$ density since the $T R^{2}$ approximate form for the LM statistic depends crucially on an assumption of conditionally normal disturbances. Therefore, in order to test whether there is evidence of autoregressive conditional kurtosis in the data, we revert to the application of a likelihood ratio test to the relevant estimated model parameters $\left(\beta_{1}\right.$ and $\beta_{2}$ in $(12 d))$.

\subsection{Extensions of the Basic GARCHK Model}

There are several natural extensions of the model given by equations (12) that arise from an examination of the comparable GARCH literature. The simplest of these would be to increase the

number of lags of the fourth power of the standardised error, $\varepsilon_{t-1}^{*} / h_{t-1}^{2}$, and of the conditional kurtosis in the same way that a $\operatorname{GARCH}(1,1)$ can be extended to a $\operatorname{GARCH}(p, q)$. Models of a higher order in the conditional kurtosis equation were considered for the applications reported below, but all additional terms could be restricted to zero under a likelihood ratio test. This 
indicates that one lag of each of the fourth power of the standardised error and of the conditional kurtosis is sufficient to capture all of the autoregressive conditional kurtosis in the data.

The second obvious extension to make to the GARCHK model is to add additional terms to the conditional variance and conditional kurtosis equations that permit the next period values of these quantities to have asymmetric responses to the signs of the innovations, in the style of Glosten $e t$ al. (1993). This could be viewed as an alternative parameterisation for the skewness in the unconditional return distribution. It would also, of course, be plausible to specify logarithmic formulations for the conditional variance and kurtosis equations in the manner of Nelson (1991), although this possibility is not pursued here. Equations (12c) and (12d) thus become

$$
\begin{aligned}
& h_{t}=\alpha_{0}+\alpha_{1} \varepsilon_{t-1}^{* 2}+\alpha_{2} h_{t-1}+\alpha_{3} I_{t-1} \varepsilon_{t-1}^{* 2} ; \\
& k_{t}=\beta_{0}+\beta_{1} \frac{\varepsilon_{t-1}^{* 4}}{h_{t}^{2}}+\beta_{2} k_{t-1}+\beta_{3} I_{t-1} \frac{\varepsilon_{t-1}^{*}}{h_{t}^{2}} ;
\end{aligned}
$$

where $I_{t-1}$ is an indicator function taking the value 1 if $\varepsilon_{t-1}{ }^{*}$ is negative and zero otherwise, with all other parts of equations (12) remaining unchanged. Clearly, it would be possible to include the asymmetry term in either the conditional variance equation or the conditional kurtosis equation or both, and we opt for the latter.

Engle et al. (1987) suggested that investors usually require compensation in the form of additional returns for taking on additional risk. Thus, a third possible intuitive extension of the GARCHK model would therefore be to allow the current return to depend on the current value of the conditional kurtosis as well as on the conditional standard deviation. Thus, equation (12a) becomes 


$$
y_{t}=\gamma_{0}+\gamma_{3} \frac{h_{t}^{1 / 2}}{\bar{y}}+\gamma_{4} k_{t}+\varepsilon_{t}^{*}
$$

where $\bar{y}$ is the mean value of $y_{t}$. Standard GARCH-M formulations use $h_{t}^{1 / 2}$ in the conditional mean. This has units of returns but $k_{t}$ is unitless, so the conditional standard deviation is divided by the mean of the returns in order to ensure that the conditional standard deviation and conditional kurtosis terms in mean have the same scale. The scaled conditional standard deviation could also be viewed as the conditional coefficient of variation. Overall, therefore, both variables in $\left(12 \mathrm{a}^{\prime}\right)$ are unitless so that the coefficients $\gamma_{3}$ and $\gamma_{4}$ both have units of returns. We do not estimate the conditional mean equation represented by (12a') in this paper, and we therefore leave a more extensive consideration of such models for future research.

\subsection{Residual Standardisation and Moment Specification Testing}

Given the model, if it is capturing all of the relevant features of the data, certain moment relationships should hold on an appropriately standardised measure of the residuals. These can be tested on their sample moments. Effectively, the tests are of non-linear restrictions on the parameters of the model given the data, and can be regarded as mis-specification tests. The relevant forms for the tests are presented in Newey (1985).

An important issue is to determine the appropriate standardised form of the residuals that should be used. It should be evident from the specification of the model of (12) that taking the residuals from the estimated GARCHK model and dividing them by the square root of the conditional variance, i.e. forming $\hat{\varepsilon}_{t}^{*} / \hat{h}_{t}^{1 / 2}$, will in general not provide an independently distributed series. Such a procedure would be inappropriate since it would ignore any conditional kurtosis that was 
present in the data. Instead, a more appropriate approach would be to take the estimated residual from the model and to divide it by the contemporaneous estimated value of the transforming variable: $\hat{\varepsilon}_{t}^{*} / \hat{\lambda}_{t}$. Under correct model specification, the result will be a standardised measure that will be an independently distributed $t$-variate with $v_{t}$ degrees of freedom. From (12f), it should also be clear that as the degrees of freedom increase towards infinity, $\lambda_{t} \rightarrow h_{t}^{1 / 2}$, and hence this new standardised residual becomes the usual measure employed to test GARCH model effectiveness, $\hat{\varepsilon}_{t}^{*} / \hat{h}_{t}^{1 / 2}$.

The quantity $\hat{z}_{t}=\hat{\varepsilon}_{t}^{*} / \hat{\lambda}_{t}$ can then be used to apply conditional moment-based specification tests of the form proposed by Newey (1985) and Nelson (1991), and in a similar vein to those examined by Harvey and Siddique (1999). The 9 orthogonality conditions examined in this paper are as follows

$\mathrm{E}\left[z_{t}\right]=0$

$\mathrm{E}\left[z_{t} \cdot z_{t-j}\right]=0$ for $j=1,2,3,4$

$\mathrm{E}\left[\left\{z_{t}^{2}-\left(v_{t} /\left(v_{t}-2\right)\right)\right\} \cdot\left\{\left(z_{t-j}^{2}-\left(v_{t-j} /\left(v_{t-j}-2\right)\right)\right\}\right]=0\right.$ for $j=1,2,3,4$

\subsection{Potential Applications of the Model}

We believe that the class of models proposed in this study could have widespread applicability in finance. The first application would be for the simulation of artificial series of asset returns that closely match the distributions and temporal profiles of actual returns series. These simulated series could be used in option pricing, or in risk measurement (e.g. for value at risk estimation). A further possible application of the approaches proposed here would be to an asset pricing model such as that of Dittmar (2002) ${ }^{\mathrm{i}}$. He examines non-linear pricing kernels for describing the cross- 
sectional variation in stock returns. His approach is applicable when investors are averse to kurtosis as well as to variance, so that returns are influenced by coskewness and cokurtosis as well as covariance.

\section{Data}

The data employed in this study comprise four financial time series obtained from Datastream International - two equity indices (US Standard and Poor's 500 and UK FTSE 100), and two bond indices (UK, US). The equity indices are total return indices where dividends have been added back to calculate the index values, while the bonds are both 10 -year maturity benchmark total return bond indices. The daily sample spans the period 2 January 1990 to 14 June 2000, implying a total of 2727 observations. The series are transformed into continuously compounded returns by taking the natural logarithms of the price relatives in the usual fashion.

Panel A of Table 1 presents summary statistics for the 4 series. Clearly, whilst all of the returns series show statistically significant evidence of leptokurtosis, the degree of unconditional skewness varies from one series to another. The FTSE 100 index returns are positively skewed but not significantly so. On the other hand, the S\&P500 returns and the US bond returns are significantly negatively skewed while the UK bond returns are negatively skewed but not significantly so. In all cases, the null hypothesis that the unconditional return distributions are normal is rejected convincingly. An application of the Ljung-Box $Q^{*}$ test using 5 lags of the returns suggest reasonable evidence of autocorrelation in the conditional mean. Closer examination of the autocorrelation and partial autocorrelation functions (not shown but available from the authors on request) suggested a first order AR (for UK equities, and both bond series) or a first-order MA (for the US equities) model as sufficient to capture this linear dependence. 
Engle's (1982) LM test for ARCH is suggestive of highly significant volatility clustering effects in each case.

\section{Results}

\subsection{Parameter Estimates}

Panel B of Table 1 gives results for estimation of Bollerslev's GARCH- $t$ model with timeinvariant kurtosis and degrees of freedom. The estimated degrees of freedom in each case can be calculated from a time-invariant version of (12e), and are 5.36, 6.45, 6.15, and 5.56 for the US equities, UK equities, US bonds and UK bonds respectively. These values together with those for the conditional variance equations are plausible and in line with the conclusions of previous research and are again indicative of the fatness of the tails of the return distributions in each case. All series except the US bond returns demonstrate strong persistence of shocks to the conditional variance, as demonstrated by the closeness of the sum of $\alpha_{1}$ and $\alpha_{2}$ to unity.

The results of estimating equations (12a), (12b), (12 $\left.\mathrm{c}^{\prime}\right),\left(12 \mathrm{~d}^{\prime}\right)$ and (12e) are presented in Table 2. This is the GARCHK model with asymmetries in the conditional mean and conditional kurtosis equations. First, it is evident that all of the conditional variance and conditional kurtosis coefficient values are positive as required. The parameter estimates concerning the conditional variance equation are entirely as expected: the persistence of shocks to volatility is high in most cases, with a higher coefficient value on the lagged conditional variance than the lagged squared error. The lagged kurtosis coefficient values are significant for all series, while the coefficients on the lagged fourth powers of the standardised error are only significant for the two equity series at 
the 5\% level, and for the US bond returns at the $10 \%$ level. The $\alpha_{1}$ coefficient has often been termed the "volatility of variance" parameter in the GARCH literature, since it measures how much the conditional variance will move around over time in response to innovations. The corresponding coefficient in the conditional kurtosis equation, $\beta_{1}$, could usefully be termed the "volatility of kurtosis" and would similarly measure the stability of the fitted conditional kurtosis in response to innovations. It is clear, for all four series, that the volatility of kurtosis is considerably greater than the corresponding volatility of variance parameters, suggesting that the fitted kurtoses are likely to be far less smooth than the fitted variances. The conditional kurtosis coefficients also show a high degree of persistence for the US bond series, although the persistence is overall far less apparent than in the corresponding conditional variance equations. In the case of the kurtosis equations, much of the persistence results from the lagged standardised innovation term than the lagged conditional kurtosis, while almost all of the persistence in the conditional variance comes from the lagged conditional variance terms.

Considering the conditional variance asymmetry parameters, $\alpha_{3}$, they are statistically significant at the $1 \%$ level for all four series. Such results are consistent with previous studies of asymmetry in volatility. The asymmetries in conditional kurtosis appear to be, from a consideration of the $t$ ratios, considerably less significant than those in conditional variance in all cases, although they do always have the expected positive sign. Thus, it can be said that negative innovations lead to higher (although not significantly so) future values of kurtosis than positive innovations of the same magnitude. The coefficient values on the asymmetry terms are bigger in the conditional kurtosis equation, ranging from 0.01 to 0.6 , indicating that the asymmetric effects are larger in magnitude, although not significant, compared with those in the conditional variance equation. 
In the context of equity markets, such asymmetries have been attributed to leverage effects - see, for example, Black (1976) or Christie (1982). The argument goes that as equity values fall, the relative weight attached to debt in a firm's capital structure rises, ceteris paribus. This induces equity holders, who bear the residual risk of the firm, to perceive the stream of future income accruing to their portfolios as being relatively more risky. An alternative view is provided by the 'volatility-feedback' hypothesis. Assuming constant dividends, if expected returns increase when stock price volatility increases, then stock prices should fall when volatility rises. Bekaert and $\mathrm{Wu}$ (2000) find strong support for the volatility feedback hypothesis rather than for the leverage effect. Whilst one cannot appeal to such explanations of leverage effects in the context of asymmetries in other financial asset return time series, there is equally no reason to suppose that such asymmetries do not exist. In the context of exchange rates, for example, it is possible that good news for one currency will have a differential impact on the perceptions of investors compared with an equivalent amount of good news for the other.

The third column from the right of Table 2 presents the maximal value of the log-likelihood function for the model with GARCHK including asymmetry terms. This can be compared with the last column of Panel B in Table 1 which gives the LLF values for the restricted model where $\alpha_{3}=0, \beta_{1}=0, \beta_{2}=0$, and $\beta_{3}=0$. The final column presents the implied likelihood ratio test value for the restriction of the GARCHK asymmetric model to have fixed kurtosis over time and no asymmetries. This statistic will follow a $\chi^{2}(4)$ under the null hypothesis, with critical value 9.48 at the 5\% level. Restrictions of the conditional kurtosis to be fixed and of no asymmetries are resoundingly rejected for both equity returns series and marginally for the US bonds, 
suggesting that there seems to be less evidence of conditional kurtosis or asymmetries in the latter, in contrast with the highly significant lagged conditional kurtosis term. This may be indicative, in the context of the complex non-linear optimisation that is required, that the overall model fit has only improved marginally upon inclusion of the $\beta_{1}$ and $\beta_{2}$ parameters as a result of a reduction in the goodness of fit of other parts of the model (although it is not apparent from the results which parts).

The last column of Table 2 gives the results of a likelihood ratio test of the restriction that the asymmetry terms in both equations are jointly zero. Such a restriction is rejected marginally for the US equity and UK bond series but not for the UK equities or US bonds. It is clear that asymmetries are very much stronger in conditional variance than conditional kurtosis, and that the results of the latter dilute those of the former in the joint test. The asymmetry terms in the conditional variance can capture the skewness in the unconditional distribution of returns, while it is less clear what the impact of asymmetry terms in the kurtosis equation would be on the unconditional distribution.

For illustration, Figure 1 plots the fitted conditional variance and conditional kurtosis obtained for the FTSE returns by estimating equations (12). Although there are periods when both the conditional variance and conditional kurtosis are high, it is evident that the model has succeeded in enabling the kurtosis to develop a different dynamic pattern. In general, the conditional kurtosis is much more stable for most of the time than the variance, but has periods where it rises very substantially. Thus the conditional kurtosis appears to be fitting to the extreme events, as one may expect. Both the conditional variance and conditional kurtosis take on high values in the 
early 1990's, but have historically low values for the 1993-96 period, before rising again towards the end of the sample.

Whilst it is easy to make ex post rationalisations of results with the benefit of hindsight, these broad patterns do appear to tie in with economic and financial events at those times. The summer of 1990 was around the time of the "Gulf crisis" following the Iraqi invasion of Kuwait, while September 1992 witnessed the Sterling ERM crisis. US inflation figures caused world-wide bond and stock market falls. The second largest ever rise in the FTSE 100 occurred on the $7^{\text {th }}$ September 1998, while it fell by 3.8\% (the largest ever fall for that index) on 4 January 2000 as a result of market fears of interest rate rises. The "Russian crisis" occurred around September 1998, and led to a global fall in equity and bond market prices, followed by a partial bounce back.

Figure 2 graphs the estimated degrees of freedom over time for the FTSE returns. The degrees of freedom never rises above 15 for the whole sample period, with an average of around 8, further indicating the fatness of the unconditional return distribution. Mirroring the conditional kurtosis, the degrees of freedom rises during the mid-1990's as the return distribution's tails thin, before falling considerably in the late 1990 's, when it spends most of its time in the 4-8 range. It is also worth noting, however, that the typical range of values that the degree of freedom parameter takes over time are higher than the fixed unconditional estimate of 6.4 from the Bollerslev $t$ model. This is suggestive that forcing the degree of freedom parameter to be fixed over time will lead to a measured tail fatness that is greater than the values it would take for most of the sample period if it were allowed to vary. Figure 3 plots the time-varying transformation parameter, $\lambda_{t}$, for the UK stock returns. The extent to which this varies over time is a measure of the extent to 
which the relationship between the conditional variance and the degrees of freedom varies over time. From (12f), it is evident that the transformation series must be non-negative. The value of $\lambda_{t}$ is also less than unity for the majority of the sample period, and so from equation (12b), the transformation results in a shrinking of the $t$-variate relative to one that follows an untransformed $t$ with $v_{t}$ degrees of freedom.

For comparison, Figures 4 to 6 replicate Figures 1 to 3, but for the US Treasury bond series. First, considering Figures 1 and 4, it is evident from examining the ranges of values of the conditional variance and conditional kurtosis that the conditional variance is typically lower for the US bond series than for the UK equities, although the higher conditional kurtosis has peaks that are higher in the bond case. Unlike the equity series, the bond returns were relatively stable in the early 1990's and at the end of our sample, while the conditional variance and conditional kurtosis rose substantially in the mid 1990's. Figure 4 scales very differently from Figure 1. In the latter case, one very large value of the kurtosis relative to its typical values has considerably changed the scaling, while there are no such extreme estimates for the US bonds. The fitted degree of freedom parameter for the US bonds (Figure 5) remains within a narrow range of values $(4,7.2)$ for the whole sample period, leading the time-varying transformation parameter to also lie within a narrower range of values than was the case for UK equities. No restrictions are placed on the model that constrain the value of the degree of freedom parameter, other than that it must be greater than 4 at all times. 


\subsection{Results of Moment-Based Specification Tests}

The results from an application of the moment-based specification tests described in Section 2.4 are given in Table 3 for the most general of the models that we consider, whose coefficient values were described in Table 2. The first five conditions in Table 3 examine the specification of the conditional mean of the standardised residuals, and the next 4 examine the conditional variance. If the model has captured all of the dynamic features of the first four moments of the returns series, none of the test statistics should be significantly different from zero. Table 3 suggests some evidence of further structure in the conditional mean for the S\&P and for both of the bond series. The covariance of the standardised residuals with their first lags are statistically significant only for the two bond series, while the covariance with the third lag is significant for the US equities. This evidence of further linear structure is not consistent with the acf and pacf results obtained from the raw (i.e. the original unstandardised) returns, but may be symptomatic of the difficulty in estimating the model also containing conditional standard deviation and conditional kurtosis terms in the mean equation. There is evidence that the dependence in the conditional variance has not been fully captured in the cases of the S\&P 500, and the US and UK bond series. Thus, overall, there still remain some features of the data that have apparently not been fully captured by the model. Harvey and Siddique (1999) found using similar moment tests that their (albeit different) model could equally not describe all of the relevant facets of the data. This should not, however, be taken as evidence that the model proposed here is less well specified than those described in previous studies. Rather, since virtually none of the studies in the GARCH literature have employed specification tests, our results may be indicative that standard GARCH $(1,1)$ models are not sufficient to fully capture the temporal dependencies in financial asset return series. Obtaining a model that is able to capture such features in their entirety is an open question for future research. 


\subsection{Comparison with the Harvey and Siddique Model}

As discussed in the introductory section of this paper, Harvey and Siddique (1999) developed a model that permits the conditional skewness to vary over time but where the conditional kurtosis is not explicitly parameterised. Thus, while we focus on the fourth moment at the expense of the third, they focus on the third moment at the expense of the fourth. Modifying their notation slightly, the model may be described by the following equations:

$$
\begin{aligned}
& y_{t}=\alpha_{0}+\alpha_{1} h_{t-1}+\varepsilon_{t}^{*} \\
& h_{t}=\beta_{0}+\beta_{1} \varepsilon_{t-1}^{* 2}+\beta_{2} h_{t-1}+\kappa h_{\mathrm{t}-1} I_{t} \\
& s_{t}=\gamma_{0}+\gamma_{1} s_{t-1}+\gamma_{2} \varepsilon_{t-1}{ }^{* 3}
\end{aligned}
$$

where $s_{t}$ denotes the conditional skewness, $I_{t}$ denotes the indicator function that sets non-positive disturbances to zero for capturing asymmetries in the conditional variance equation. Note that their indicator function is defined in the opposite way to that used by Glosten et al. Harvey and Siddique do not consider asymmetries in the skewness equation (13c). The results from estimation of their model are presented in Table 4.

As the results show, the intercept and lagged conditional skewness terms, $\gamma_{1}$, are significant and negative for all four series, but the lagged cubed disturbance parameter is significant only for the US stock series; this result is identical to that of Harvey and Siddique. However, the magnitudes of the parameters are different for the US stock series due to the considerably different sample periods in our paper compared with theirs.

\section{Conclusions}


This paper has proposed and estimated a model for conditional kurtosis. The model is based on the approach of Bollerslev (1987), but its novelty lies in its ability to allow the conditional kurtosis to develop in a fashion that is not fixed to the conditional variance. This occurs via a time-varying degree of freedom parameter. The model was applied to a set of four financial asset return time series, and the results indicate strong evidence for the presence of "GARCH-style" dependence in the conditional kurtosis, suggesting the presence of a hitherto unexplored phenomenon in such series.

Several extensions to the basic model were proposed, including considerations of asymmetries in the relationship between the sign of the innovations and the size of the next period conditional kurtosis, and of a possible feedback from the kurtosis to the returns. Evidence for these relationships appeared to be weaker in the context of conditional kurtosis than was the case for the conditional variance.

The research described above could be usefully extended in a number of directions. First, the models proposed could be used to produce conditional kurtosis forecasts. These predictions may be useful - for example in the pricing of some classes of financial asset, such as options on options that require estimates of the "variance of a variance". This quantity could be obtained from the forecasts of conditional kurtosis, and the option prices obtained compared with those from simpler approaches. Second, it may be the case that models allowing for dynamic higher moments can better describe the distributional properties of financial asset returns, especially when measured at high frequency, than less complex models that do not. Third, models containing feedback terms from the conditional variance and kurtosis to the conditional mean ("GARCHK-M" models) may, when appropriately formulated, be used to obtain separate 
estimates of the market-required risk premia for accepting variance (or standard deviation) and kurtosis risk. GARCHK-M models could then be used in portfolio construction or in investment performance appraisal to evaluate whether the trade-off between mean, variance, and kurtosis that is implicit from the series of returns to the chosen portfolio was an optimal one given the market-required returns for each type of risk. Finally, the development of a multivariate version of the model should shed light on the co-relationships between the moments of each series employed in the system, which is likely to be of relevance in the context of portfolio construction or financial risk management.

\section{References}

Bekaert, G., Erb, C.B., Harvey, C., and Viskanta, T.E. (1998). Distributional Characteristics of Emerging Market Returns and Asset Allocation Journal of Portfolio Management Winter, 102-116.

Bekaert, G. and Wu, G. (2000). Asymmetric Volatility and Risk in Equity Markets Review of Financial Studies 13, 1-42.

Black, F. (1976). Studies in price volatility changes, Proceedings of the 1976 Meeting of the Business and Economics Statistics Section American Statistical Association, 177-181.

Bollerslev, T. (1986). Generalized autoregressive conditional heteroscedasticity Journal of Econometrics $31,307-327$.

Bollerslev, T. (1987). A conditionally heteroskedastic time series model for speculative prices and rates of return Review of Economics and Statistics, 69, 542 - 547. 
Bond, S.A. (2001). A review of asymmetric conditional density functions in autoregressive conditional heteroscedasticity models in Knight, J. and Satchell, S. (eds.) Return Distributions in Finance Butterworth-Heinemann, Oxford, UK.

Christie, A. (1982). The stochastic behaviour of common stock variance: Value, leverage and interest rate effects Journal of Financial Economics, 10, 407-432.

Chunhachinda, P., Dandapani, K., Hamid, S., and Prakash, A. (1997). Portfolio Selection and Skewness: Evidence from International Stock Markets Journal of Banking and Finance 21, 143-167.

Engle, R.F. (1982). Autoregressive conditional heteroscedasticity with estimates of the variance of United Kingdom inflation. Econometrica 50, 987-1006.

Engle, R.F. and Bollerslev, T. (1986). Modelling the persistence of conditional variances. Econometric Reviews 5, 1-50.

Engle, R.F., Lilien, D.M., Robins, R.P. (1987). Estimating Time Varying Risk Premia in the Term Structure: The ARCH-M Model Econometrica 55(2), 391-407.

Fernandez, C. and Steel, M.F.J. (1998). On Bayesian Modelling of Fat Tails and Skewness Journal of the American Statistical Association 93, 359-371.

Glosten, L.R., Jagannathan, R. and Runkle, D.E. (1993). On the Relation Between the Expected Value and the Volatility of the Nominal Excess Return on Stocks The Journal of Finance 48(5), 1779-1801. 
Hansen, B.E. (1994). Autoregressive Conditional Density Estimation International Economic Review 35, 705-730.

Harvey, C.R. and Siddique, A. (2000). Conditional Skewness in Asset Pricing Tests Journal of Finance $55,1263-1295$.

Harvey, C.R. and Siddique, A. (1999). Autoregressive Conditional Skewness Journal of Financial and Quantitative Analysis 34, 465-487.

Johansen, A., and Sornette, D. (1999). Critical Crashes Risk 12(1), 91-95.

Jondeau, E. and Rockinger, M. (2000). Conditional Volatility, Skewness and Kurtosis: Existence and Persistence Mimeo. Banque de France and HEC School of Management.

Lambert, P. and Laurent, S. (2001). Modelling Financial Time Series using GARCH-type Models and a Skewed Student Density Mimeo. Université Catholique de Lovain.

Nelson, D. (1996). Asymptotic Filtering Theory for Multivariate ARCH Models Journal of Econometrics $71,1-47$.

Nelson, D.B. (1991). Conditional Heteroskedasticity in Asset Returns: A New Approach Econometrica 59(2), 347-370

Newey, W. (1985). Generalised Method of Moments Specification Testing Journal of Econometrics 29, 229-256. 
Premaratne, G. and Bera, A.K. (2001). Modelling Asymmetry and Excess Kurtosis in Stock Return Data Mimeo. Department of Economics, University of Illinois.

Rockinger, M and Jondeau, E. (2001). Conditional Dependency of Financial Series: An Application of Copulas Persistence Mimeo. Banque de France and HEC School of Management.

Scott, R. and P. Horvath (1980). On the Direction of Preference for Moments of Higher Order Than the Variance, Journal of Finance, 35, 915-919.

Theodossiou, P. (1998). Financial Data and the Skewed Generalised T Distribution Management Science $12,1650-1661$. 
Table 1: Summary Statistics and Model Estimates for GARCH with fixed degrees of freedom Panel A: Summary Statistics

\begin{tabular}{lccccccc}
\hline & Mean & Variance & Skewness & $\begin{array}{c}\text { Kurtosis } \\
\text { (excess) }\end{array}$ & $\begin{array}{c}\text { BJ }^{*} \\
\text { Normality }\end{array}$ & $\begin{array}{c}\text { Ljung-Box } \\
\text { Q*(5) }\end{array}$ & ARCH(5) \\
\hline Equities & & & & & & & \\
S\&P500 & 0.022 & 0.159 & $-0.345^{* *}$ & $5.415^{* *}$ & $3385.130^{* *}$ & $12.754^{*}$ & $255.253^{* *}$ \\
FTSE 100 & 0.015 & 0.125 & 0.014 & $2.826^{* *}$ & $906.954^{* *}$ & $29.024^{* *}$ & $274.823^{* *}$ \\
& & & & & & & \\
Bonds & & & & & & & \\
US & 0.002 & 0.053 & $-0.305^{* *}$ & $1.957^{* *}$ & $477.062^{* *}$ & $19.961^{* *}$ & $16.649^{* *}$ \\
UK & 0.007 & 0.050 & -0.020 & $3.736^{* *}$ & $1585.630^{* *}$ & $12.226^{*}$ & $78.74^{* *}$ \\
\hline
\end{tabular}

Panel B: Estimates for GARCH with fixed degrees of freedom

$$
\begin{aligned}
& y_{t}=\gamma_{0}+\gamma_{1} y_{t-1}+\gamma_{2} \varepsilon_{t-1}{ }^{*}+\varepsilon_{t}^{*} \\
& \varepsilon_{t}^{*}=\lambda_{t} \varepsilon_{t}, \varepsilon_{t} \sim t_{v} \\
& h_{t}=\alpha_{0}+\alpha_{1} \varepsilon_{t-1}^{* 2}+\alpha_{2} h_{t-1} \\
& k_{t}=\beta_{0}
\end{aligned}
$$

\begin{tabular}{|c|c|c|c|c|c|c|c|c|}
\hline & $\gamma_{0}$ & $\gamma_{1}$ & $\gamma_{2}$ & $\alpha_{0}$ & $\alpha_{1}$ & $\alpha_{2}$ & $\beta_{0}$ & LLF \\
\hline \multicolumn{9}{|l|}{ Equities } \\
\hline S\&P500 & $\begin{array}{c}0.060 \\
(0.014)^{* *}\end{array}$ & - & $\begin{array}{c}0.034 \\
(0.024)\end{array}$ & $\begin{array}{c}0.043 \\
(0.005)^{* *}\end{array}$ & $\begin{array}{c}0.286 \\
(0.015)^{* *}\end{array}$ & $\begin{array}{c}0.730 \\
(0.008)^{* *}\end{array}$ & $\begin{array}{c}7.404 \\
(2.126)^{* *}\end{array}$ & -1736.96 \\
\hline FTSE 100 & $\begin{array}{c}0.031 \\
(0.012)^{*}\end{array}$ & $\begin{array}{c}0.063 \\
(0.022)^{* *}\end{array}$ & - & $\begin{array}{c}0.057 \\
(0.004)^{* *}\end{array}$ & $\begin{array}{c}0.273 \\
(0.014)^{* *}\end{array}$ & $\begin{array}{c}0.711 \\
(0.009)^{* *}\end{array}$ & $\begin{array}{c}5.449 \\
(1.319)^{* *}\end{array}$ & -1517.63 \\
\hline \multicolumn{9}{|l|}{ Bonds } \\
\hline US & $\begin{array}{l}0.0116 \\
(0.009)\end{array}$ & $\begin{array}{c}0.036 \\
(0.019)\end{array}$ & - & $\begin{array}{c}0.172 \\
(0.006)^{* *}\end{array}$ & $\begin{array}{c}0.019 \\
(0.012)\end{array}$ & $\begin{array}{c}0.384 \\
(0.020)^{* *}\end{array}$ & $\begin{array}{c}5.791 \\
(0.799)^{* *}\end{array}$ & -495.74 \\
\hline UK & $\begin{array}{c}0.0249 \\
(0.008)^{* *}\end{array}$ & $\begin{array}{c}0.043 \\
(0.019)^{*}\end{array}$ & - & $\begin{array}{c}0.004 \\
(0.001)^{* *}\end{array}$ & $\begin{array}{c}0.045 \\
(0.002)^{* *}\end{array}$ & $\begin{array}{c}0.938 \\
(0.002)^{* *}\end{array}$ & $\begin{array}{c}6.846 \\
(0.683)^{* *}\end{array}$ & -279.36 \\
\hline
\end{tabular}

Notes: Asymptotic standard errors are shown in parentheses. * The Bera-Jarque (BJ) normality test is asymptotically distributed as a $\chi^{2}(2)$ variate under the null of normality while the Ljung-Box and ARCH tests are asymptotically distributed as $\chi^{2}(5)$ variates under their respective null hypotheses. The $5 \% \chi^{2}(2)$ and $\chi^{2}(5)$ critical values are respectively 5.991 and 11.071 , and at the $1 \%$ level, the critical values are 9.210 and 15.086 respectively. $*$ and $* *$ denote significance at the 5\% and $1 \%$ levels respectively; LLF denotes the maximal value of the log-likelihood function. 
Table 2: An Asymmetric Model with Conditional Variance \& Kurtosis

Model: $y_{t}=\gamma_{0}+\gamma_{1} y_{t-1}+\gamma_{2} \varepsilon_{t-1}{ }^{*}+\varepsilon_{t}^{*}, \varepsilon_{t}^{*}=\lambda_{t} \varepsilon_{t}, \varepsilon_{t} \sim t_{v_{t}}, h_{t}=\alpha_{0}+\alpha_{1} \varepsilon_{t-1}^{* 2}+\alpha_{2} h_{t-1}+\alpha_{3} I_{t-1} \varepsilon_{t-1}^{* 2}, k_{t}=\beta_{0}+\beta_{1} \frac{\varepsilon_{t-1}^{*}}{h_{t-1}^{2}}+\beta_{2} k_{t-1}+\beta_{3} I_{t-1} \frac{\varepsilon_{t-1}^{*}}{h_{t-1}^{2}}$

$\gamma_{0}$

$\begin{array}{lll}\gamma_{1} & \gamma_{2} & \alpha_{0}\end{array}$

$\alpha_{1}$

$\alpha_{2}$

$\alpha_{3}$

$\beta_{0}$

$\beta_{1}$

$\beta_{2} \quad \beta_{3}$

$\beta_{3}$

LLF

$$
\begin{array}{cc}
\text { LR: } & \text { LR: } \\
\alpha_{3}=0 & \alpha_{3} \beta_{1}, \beta_{2}, \\
\beta_{3}=0 & \beta_{3}=0
\end{array}
$$

\begin{tabular}{|c|c|c|c|c|c|c|c|c|c|c|c|c|c|c|}
\hline \multicolumn{15}{|l|}{ Equities } \\
\hline S\&P500 & $\begin{array}{c}0.048 \\
(0.013)^{* *}\end{array}$ & - & $\begin{array}{c}0.031 \\
(0.023)\end{array}$ & $\begin{array}{c}0.025 \\
(0.003)^{* *}\end{array}$ & $\begin{array}{c}0.081 \\
(0.008)^{* *}\end{array}$ & $\begin{array}{c}0.816 \\
(0.005)^{* *}\end{array}$ & $\begin{array}{c}0.164 \\
(0.016)^{* *}\end{array}$ & $\begin{array}{c}5.348 \\
(0.566)^{* *}\end{array}$ & $\begin{array}{c}0.419 \\
(0.151)^{* *}\end{array}$ & $\begin{array}{c}0.209 \\
(0.100)^{*}\end{array}$ & $\begin{array}{c}0.606 \\
(0.318)\end{array}$ & -1685.66 & $7.00 * *$ & $102.6^{* * *}$ \\
\hline FTSE 100 & $\begin{array}{c}0.027 \\
(0.012)^{*}\end{array}$ & $\begin{array}{c}0.079 \\
(0.020)^{* *}\end{array}$ & - & $\begin{array}{c}0.026 \\
(0.002)^{* *}\end{array}$ & $\begin{array}{c}0.080 \\
(0.006)^{* *}\end{array}$ & $\begin{array}{c}0.819 \\
(0.005)^{* *}\end{array}$ & $\begin{array}{c}0.140 \\
(0.014)^{* *}\end{array}$ & $\begin{array}{c}2.239 \\
(0.161)^{* *}\end{array}$ & $\begin{array}{c}0.444 \\
(0.136)\end{array}$ & $\begin{array}{c}0.370 \\
(0.036)^{* *}\end{array}$ & $\begin{array}{c}0.500 \\
(0.330)\end{array}$ & -1469.76 & 2.84 & $47.9 * *$ \\
\hline \multicolumn{15}{|l|}{ Bonds } \\
\hline US & $\begin{array}{c}0.008 \\
(0.009)\end{array}$ & $\begin{array}{c}0.039 \\
(0.019)^{* *}\end{array}$ & - & $\begin{array}{c}0.062 \\
(0.002)^{* *}\end{array}$ & $\begin{array}{c}0.013 \\
(0.009)\end{array}$ & $\begin{array}{c}0.729 \\
(0.009)^{* *}\end{array}$ & $\begin{array}{c}0.098 \\
(0.018)^{* *}\end{array}$ & $\begin{array}{c}4.640 \\
(0.912)^{* *}\end{array}$ & $\begin{array}{c}0.356 \\
(0.425)\end{array}$ & $\begin{array}{c}0.139 \\
(0.125)\end{array}$ & $\begin{array}{c}0.291 \\
(0.865)\end{array}$ & -484.35 & 3.22 & $11.4^{*}$ \\
\hline UK & $\begin{array}{c}0.017 \\
(0.008)^{*}\end{array}$ & $\begin{array}{c}0.054 \\
(0.018)^{* *}\end{array}$ & - & $\begin{array}{c}0.005 \\
(0.001)^{* *}\end{array}$ & $\begin{array}{c}0.024 \\
(0.002)^{* *}\end{array}$ & $\begin{array}{c}0.937 \\
(0.002)\end{array}$ & $\begin{array}{c}0.045 \\
(0.005)^{* *}\end{array}$ & $\begin{array}{c}4.610 \\
(2.000)^{*}\end{array}$ & $\begin{array}{c}0.021 \\
(0.767)\end{array}$ & $\begin{array}{c}0.519 \\
(0.149)^{* *}\end{array}$ & $\begin{array}{c}0.012 \\
(1.890)\end{array}$ & -274.61 & $6.22 *$ & 9.5 \\
\hline
\end{tabular}

Notes: Standard errors are shown in parentheses. $*$ and $* *$ denote significance at the $5 \%$ and $1 \%$ levels respectively. LLF denotes the maximal value of the log-likelihood function, while LR denotes the value of the likelihood ratio test statistic. The $\chi^{2}(2)$ critical values are 5.99 and 9.21 at the $5 \%$ and $1 \%$ levels respectively, and the $\chi^{2}(4)$ critical values are 9.48 and 13.28 at the 5\% and $1 \%$ levels respectively. 
Table 3: Moment Specification Tests

\begin{tabular}{lcccc}
\hline Orthogonality Conditions & US & UK & US & UK \\
& S\&P500 & FTSE100 & T-Bond & T-Bond \\
\hline 1. $\mathrm{E}\left[z_{t}\right]=0$ & -0.012 & 0.010 & -0.016 & -0.004 \\
& $(1.621)$ & $(1.910)$ & $(3.825)$ & $(0.194)$ \\
2. $\mathrm{E}\left[z_{t} \cdot z_{t-1}\right]=0$ & $0.076^{* *}$ & 0.018 & $0.028^{* *}$ & $0.038^{* *}$ \\
3. $\mathrm{E}\left[z_{t} \cdot z_{t-2}\right]=0$ & $(35.045)$ & $(2.381)$ & $(7.457)$ & $(7.249)$ \\
& 0.037 & 0.026 & 0.029 & 0.038 \\
4. $\mathrm{E}\left[z_{t} \cdot z_{t-3}\right]=0$ & $(1.580)$ & $(0.098)$ & $(0.965)$ & $(2.00)$ \\
& $-0.087 * *$ & -0.014 & -0.052 & -0.029 \\
5. $\mathrm{E}\left[z_{t} \cdot z_{t-4}\right]=0$ & $(9.218)$ & $(0.281)$ & $(3.264)$ & $(1.022)$ \\
& -0.044 & 0.023 & -0.053 & 0.033 \\
6. $\mathrm{E}\left[\left(z_{t}^{2}-\left(v_{t} /\left(v_{t}-2\right)\right)\right)\left(z_{t-1}^{2}-\left(v_{t-1} /\left(v_{t-1}-2\right)\right)\right)\right]=0$ & $(2.282)$ & $(0.837)$ & $(3.173)$ & $(1.267)$ \\
7. $\mathrm{E}\left[\left(z_{t}^{2}-\left(v_{t} /\left(v_{t}-2\right)\right)\right)\left(z_{t-2}{ }^{2}-\left(v_{t-2} /\left(v_{t-2}-2\right)\right)\right)\right]=0$ & $-0.643 * *$ & -0.277 & $-0.443^{* *}$ & -0.146 \\
8. $\mathrm{E}\left[\left(z_{t}^{2}-\left(v_{t} /\left(v_{t}-2\right)\right)\right)\left(z_{t-3}{ }^{2}-\left(v_{t-3} /\left(v_{t-3}-2\right)\right)\right)\right]=0$ & $(19.298)$ & $(3.317)$ & $(21.188)$ & $(0.419)$ \\
& -0.108 & $-0.231 *$ & -0.066 & $-0.336^{* *}$ \\
9. $\mathrm{E}\left[\left(z_{t}^{2}-\left(v_{t} /\left(v_{t}-2\right)\right)\right)\left(z_{t-4}{ }^{2}-\left(v_{t-4} /\left(v_{t-4}-2\right)\right)\right)\right]=0$ & $(0.222)$ & $(4.761)$ & $(0.312)$ & $(8.739)$ \\
& $-0.453^{* *}$ & -0.111 & -0.160 & -0.066 \\
& $(9.421)$ & $(0.924)$ & $(1.169)$ & $(0.176)$ \\
\end{tabular}

Notes: Specification tests are applied to the transformed residuals from the conditional kurtosis model with asymmetries in the conditional variance and kurtosis equations and variance and kurtosis terms in the conditional mean. Orthogonality conditions are based on $\hat{z}_{t}=\hat{\varepsilon}_{t}{ }^{*} / \hat{\lambda}_{t} *$ and $* *$ denote significance at the $5 \%$ and $1 \%$ levels respectively. Cell entries refer to sample averages with test statistics in parentheses. The latter follow a $\chi^{2}(1)$ distribution under the null hypothesis in each case. 
Table 4: Autoregressive Conditional Skewness Model Parameter Estimates and $t$-ratios

Model: $y_{t}=\alpha_{0}+\alpha_{1} h_{t-1}+\varepsilon_{t}^{*}$

$$
h_{t}=\alpha_{0}+\alpha_{1} \varepsilon_{t-1}^{* 2}+\alpha_{2} h_{t-1}+\kappa h_{\mathrm{t}-1} I_{t}
$$

$s_{t}=\gamma_{0}+\gamma_{1} s_{t-1}+\gamma_{2} \varepsilon_{t-1}{ }^{* 3}$

\begin{tabular}{ccccccccccc}
\hline Series & $\alpha_{0}$ & $\alpha_{1}$ & $\beta_{0}$ & $\beta_{1}$ & $\beta_{2}$ & $\kappa$ & $\gamma_{0}$ & $\gamma_{1}$ \\
\hline S\&P 500 & 0.022 & -0.054 & 0.027 & 0.750 & 0.117 & -0.096 & -0.010 & -0.370 & 0.016 \\
& $(2.437)$ & $(-3.333)$ & $(2.726)$ & $(11.09)$ & $(4.321)$ & $(-3.417)$ & $(2.551)$ & $(-4.546)$ & $(6.563)$ \\
FTSE 100 & 0.031 & -0.075 & 0.070 & 0.404 & 0.364 & -0.232 & -0.016 & -0.984 & -0.001 \\
& $(1.607)$ & $(-1.032)$ & $(15.717)$ & $(9.145)$ & $(5.278)$ & $(-2.484)$ & $(3.444)$ & $(-46.483)$ & $(-1.204)$ \\
US bonds & 0.0984 & -0.384 & 0.053 & 0.434 & 0.000 & -0.052 & -0.023 & -0.740 & -0.008 \\
& $(2.521)$ & $-1.964)$ & $(6.861)$ & $(6.835)$ & $(0.000)$ & $(-1.466)$ & $(-2.518)$ & $(-8.382)$ & $(-1.160)$ \\
UK bonds & 0.001 & 0.035 & 0.017 & 0.577 & 0.021 & -0.140 & -0.001 & -0.648 & -0.001 \\
& $(0.234)$ & $(0.481)$ & $(302.03)$ & $(29.238)$ & $(5.556)$ & $(-2.630)$ & $(-262.20)$ & $(-4.093)$ & $(-0.138)$ \\
\hline
\end{tabular}

Note: $t$-ratios in parentheses. 
Figure 1: Fitted Conditional Heteroscedasticity and Conditional Kurtosis over Time for UK Stock Returns

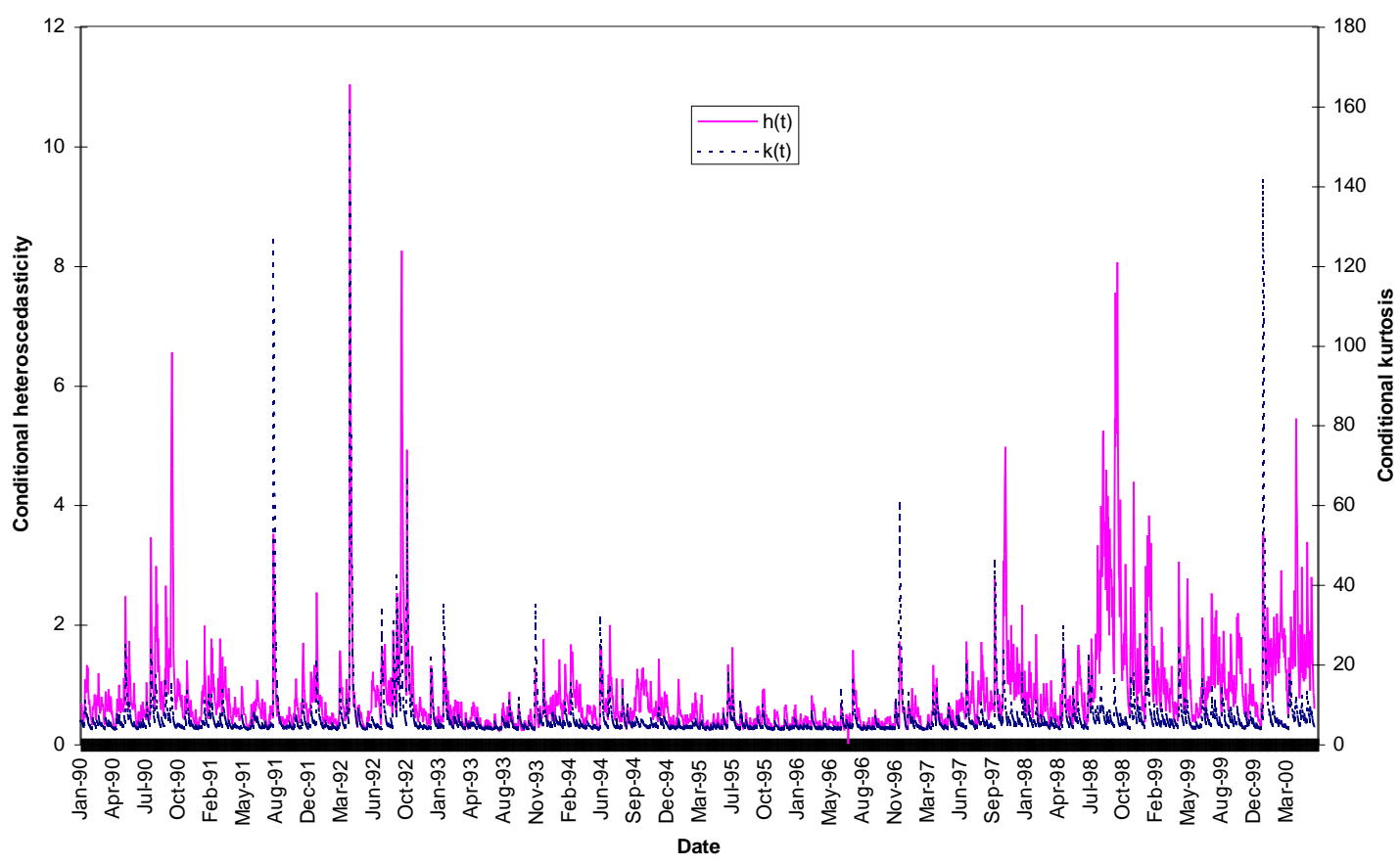

Figure 2: Estimated Degrees of Freedom over Time for UK Stock Returns

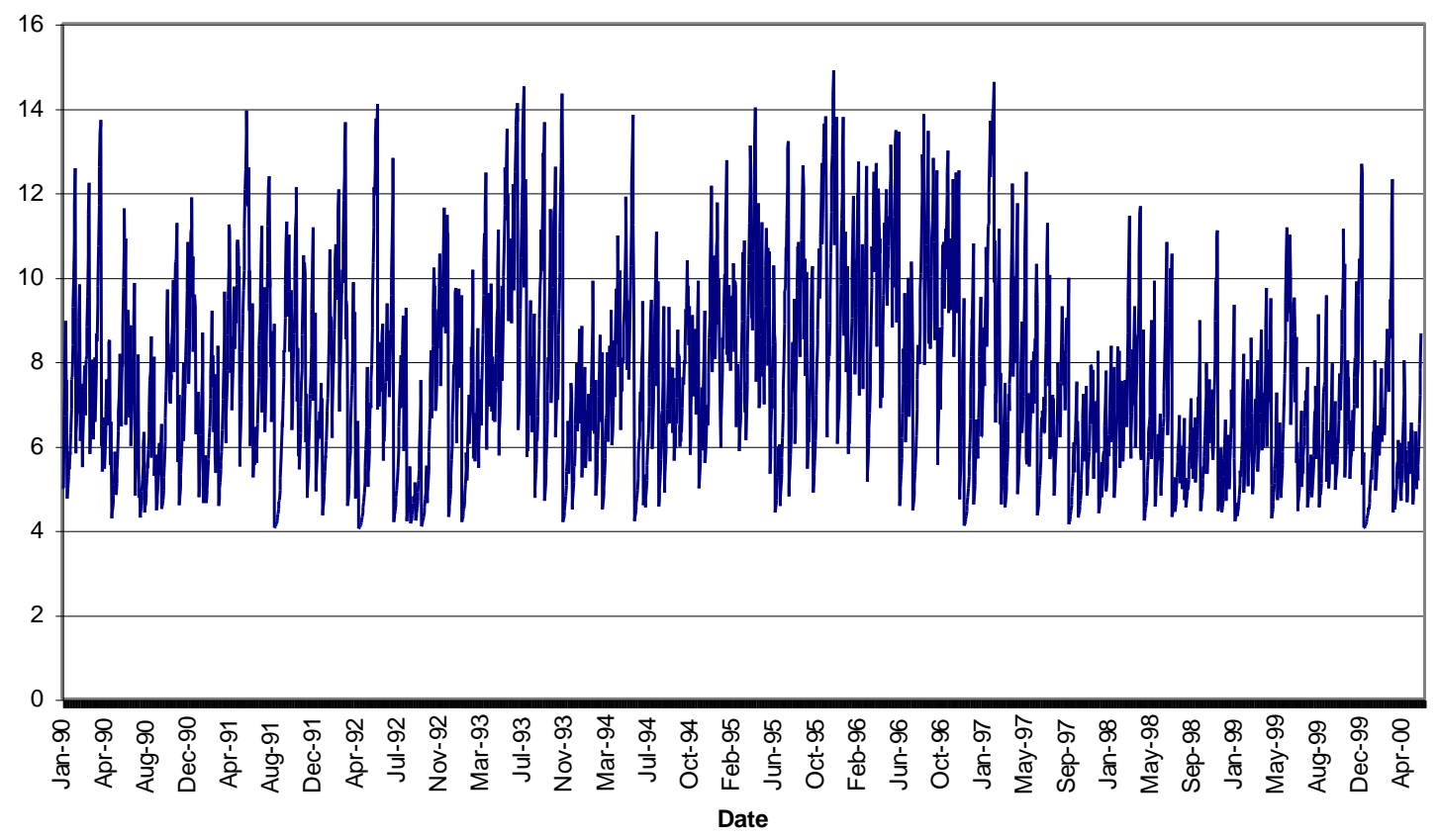


Figure 3: Plot of Transformation Series, $\lambda_{t}$ for UK Stock Returns

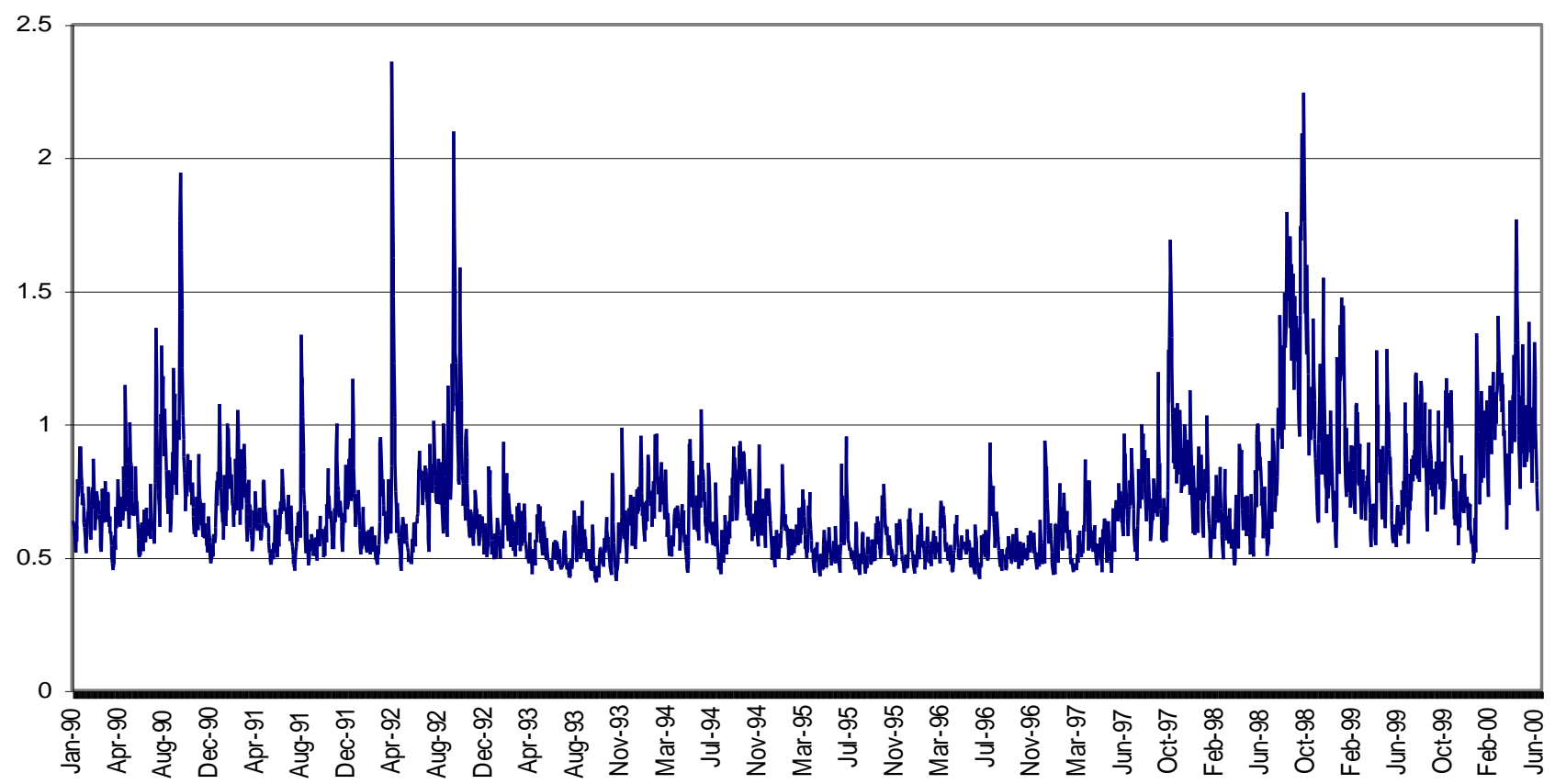

Figure 4: Fitted Conditional Heteroscedasticity and Conditional Kurtosis over Time for US T-Bonds

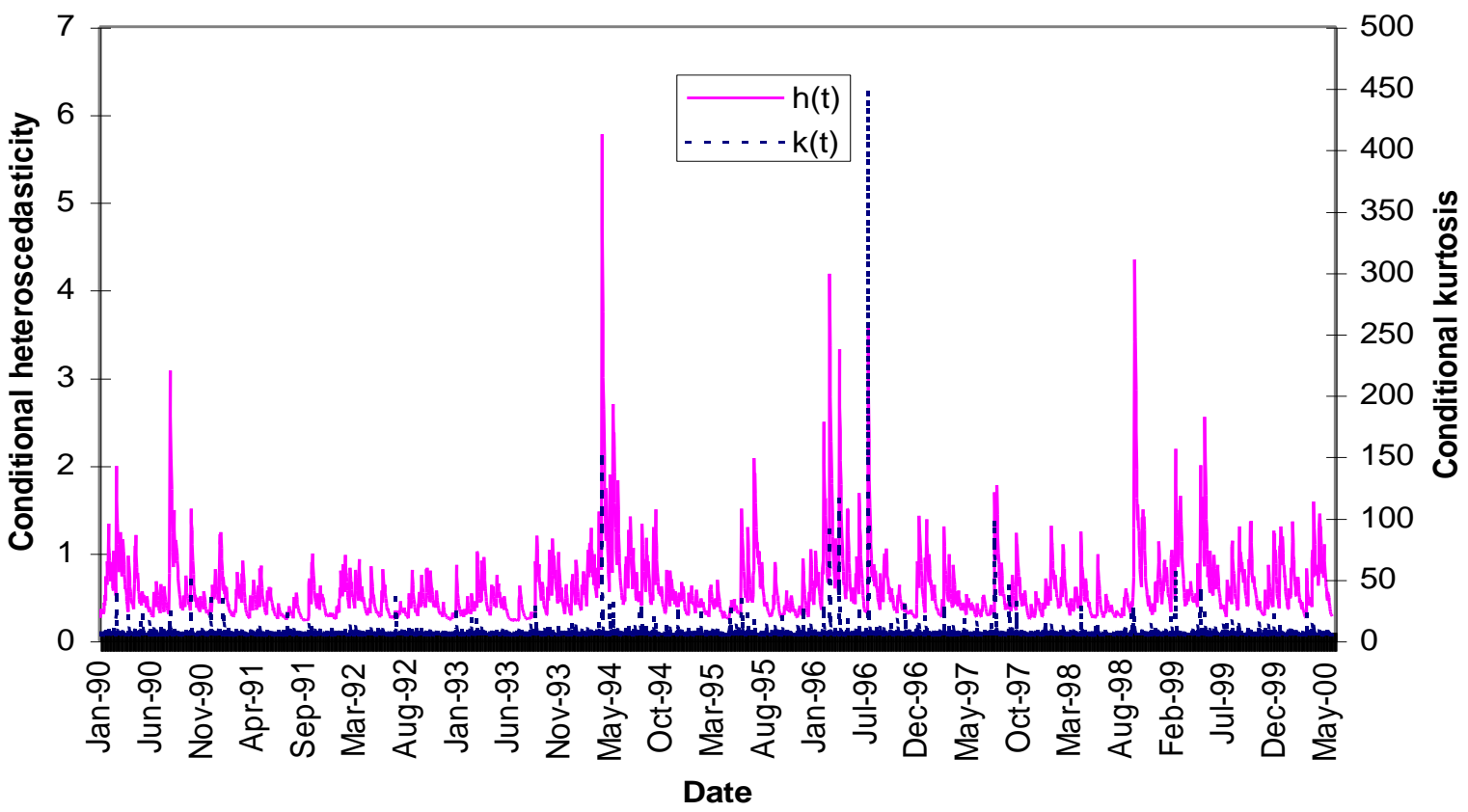


Figure 5: Estimated Degrees of Freedom over Time for US T-bonds

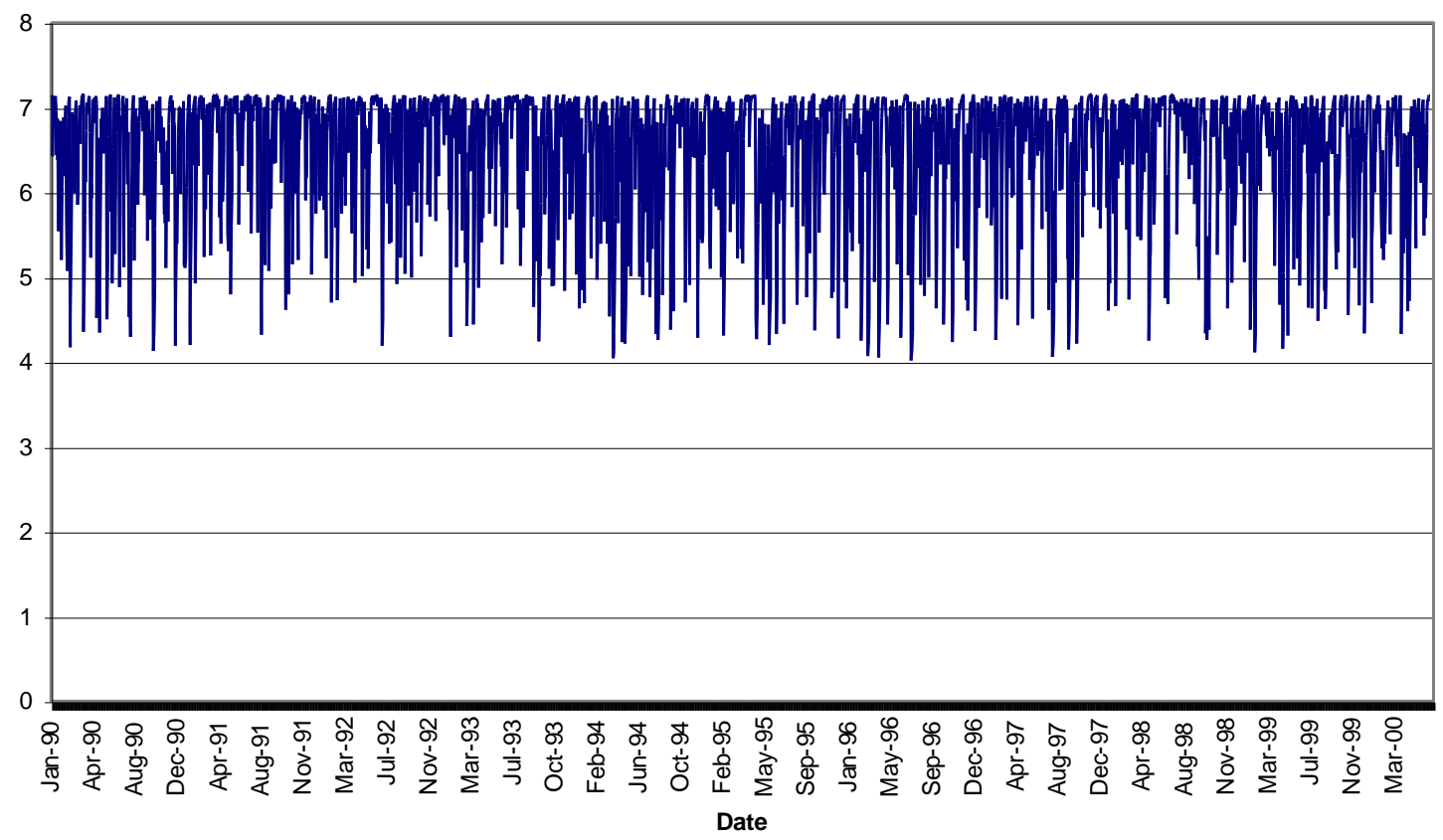

Figure 6: Plot of Transformation Series, $\lambda_{t}$ for US T-Bonds

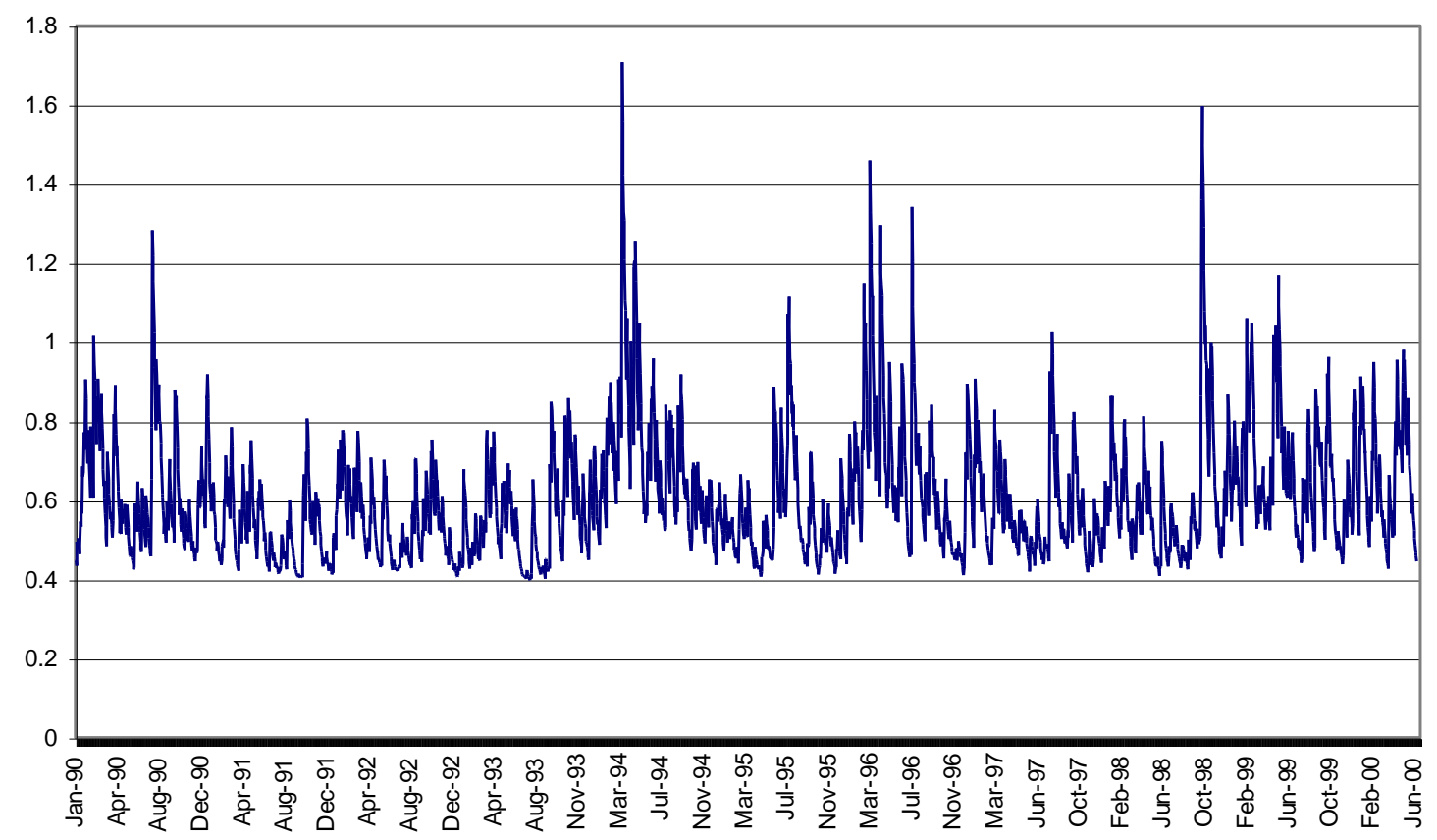


${ }^{\mathrm{i}}$ We thank an anonymous referee for suggesting this possibility. 